\title{
Future of Clinical Trials in Nepal
}

\author{
Sathian B1
}

${ }^{1}$ Assistant Professor, Department of Community Medicine, Manipal College of Medical Sciences, Pokhara, Nepal

\section{Editorial}

\section{Corresponding Author:}

Dr. Brijesh Sathian, Assistant Professor, Department of Community Medicine, Manipal College of Medical Sciences, Pokhara, Nepal.

Email:drsathian@gmail.com

A workshop on clinical trials and systematic reviews, held in Nepal recently, motivated me to think about the future of clinical trials conducted in this region. There was an intense discussion between the international resource person on one side and the clinicians and health research workers on the other. The former stated that the research hierarchy starts from a case report, which is followed by case series and ends with experimental studies. The latter group disagreed with this and tried to prove that case reports are more valuable. I agree with this opinion, case reports are better than poor conducted clinical trials. I have not worked in many clinical trials in Nepal, because there was sufficient information to suggest that trials were unnecessary. There was the potential to harm participants by either the use of or withholding the intervention and the participants would not have any benefit directly from the research. These trials were running without proper funding, patient health insurance, written consent stating the harmfulness, randomisation, study design or follow up. None of the study statisticians were included in the planning and conducting part. I still doubt whether the accountable number of number of medical statisticians are well trained in clinical trials and systematic review. Most of the researchers were not aware of clinical trial registry and Declaration of Helsinki. It is not known who will pay compensation, once the patient comes to know of any complications that occurred during the clinical trial. Researchers do not know the importance of ethical issues in clinical trials. They believe clinical trials are like cohort studies. National and international journals should make sure that researchers do not copy and paste the material and methods part of of a similar well conducted study without maintaining and executing similar protocols. Journal editors should set up several initiatives that will improve the quality of reporting of randomised trials, with prime importance given to the transparency of research in general and by emphasising the importance of protocols. They should offer to review protocols to improve trial quality and lessen publication bias. They should consider submissions of randomised trials only if registered and accompanied by a protocol, which will be sent with the manuscript to peer reviewers. Journals should prevent this by constructing an editorial board including qualified editors from developed and developing countries in the editorial board. Nowadays the output-and rewards-of research are based almost entirely on published papers in scientific journals. Scientists in low-income and middle-income settings would like to conduct research for their populations according to their own concerns. They want to be in the frontlines of national and global communications about their country's experiences. I recommend that properly trained clinical trial experts from developed countries should conduct some workshops on the design, conduct and ethical issues in clinical trials to terminate the research misconduct in developing countries.

Clinical trials are commonly carried out these days and it forms important sources of information for the rational use of drugs. The issues of informed consent, patient autonomy, liability for injury, financial inducements and the doctorpatient relationship are important ethical issues which can be explored through clinical trials ${ }^{1}$. Clinical trials do, in general, tell us a good deal about how well a drug works for a defined disease and what potential harm it may cause. They provide only limited information for larger populations with different characteristics from the trial group age, gender, state of health, ethnic origin, and so on. If a drug were later tested on many more people, or on people of different ethnic origin, for example, there might be very different results. 


\section{Monitoring of clinical trials}

In most clinical trials patients are entered one at a time, so that their responses to treatment are also observed sequentially. In this article authors would like to describe the use, rationale and suggestions for of monitoring the trials while the trial is in progress ${ }^{2-8}$.

\section{Reasons for monitoring}

\section{Protocol compliance}

Inspection of each patient's result provides an immediate awareness of any deviations from the intended procedure. If so, the investigators are forewarned about the protocol compliance. In case of general difficulties with compliance it may be necessary to make alterations to the protocol.

\section{Adverse effects}

It is necessary to monitor the side effects, particularly severe toxic reactions to a new therapy, so that prompt action can be taken. Investigators need to be warned to look out for such events in future patients. It is also necessary to define dose modifications.

\section{Data processing}

It is essential to organise the data for statistical analysis from the very beginning. One should provide an organised check on the progress of the trial from the early stages.

\section{General information}

Some general results on how the trial is progressing are necessary to maintain interest and to satisfy the natural curiosity among investigators.

\section{Treatment comparisons}

The primary reason for monitoring the data is for treatment differences which are sufficiently convincing and important to stop or change the trial. Primary reason for monitoring such treatment differences are to consider the ethical concern to prevent any patient in the trial from receiving unethical treatment. The workers should avoid such problems while undertaking interim analyses like organising and interpreting the interim results.

\section{Solutions to these problems}

\section{Measures of patient response}

You should decide which patient outcome is to be of value in interim comparisons. If a large number of variables are used there might be a problem of interpreting multiple outcomes.

\section{Data preparation}

Interim analyses should be based on data that are correct, complete and up to date. Delay in the processing of patient evaluation forms should not distort the validity of the analysis.

\section{Feasibility of interim analyses}

Interim analyses on inadequate sample size may be impractical or misleading. If the treatment duration is short and the time lag between patient entry and observance of outcome is long relative to the total period of patient accrual then there will be insufficient data for interim analyses.

\section{The decision making process}

The decision to stop or continue a trial should not be considered a purely statistical exercise. The decision should be considered in the light of current knowledge, practical aspects of therapy, degree of enthusiasm for the trial and future research ideas.

\section{Confidentiality of interim results}

Results of the interim analyses shown to an investigator could change his outlook and future participation. Hence some secrecy over interim results is necessary. In a multicenter trial, a monitoring committee may be supplied with full interim results to be interpreted confidentially.

\section{The extent of each analysis}

Interim analyses need not be elaborate since it is used only to decide whether the trial should continue in its present form. Required analyses are crude comparison on major end points, In case of results approaching statistical significance, some key prognostic factors can be looked at.

\section{Frequency of analysis}

Continuous monitoring of the accumulating data and immediate decision to stop the trial are rarely feasible. It is more effective and reliable to make a special effort to analyze interim results at periodic intervals.

\section{Statistical rules for stopping the trials}

One needs to decide in advance the magnitude of treatment difference and also the statistical significance to make a decision on stopping the trial. For a sequence of interim analysis one must set a more stringent significance level than $\mathrm{P}<0.05$.

Forming a committee to monitor the clinical trial may be warranted for a small fraction of exploratory trials. Such a panel could consist of both trial investigators and outside experts.

\section{Conflict of Interests}

The author has no conflict of interest arising from the study.

\section{References}

1. P. Ravi Shankar. Rational Use of Medicines, Pharmacology and the Medical Humanities. Siriraj Med J 2008;60:159-160. E-journal: http://www.sirirajmedj.com

2. WHO. Safety monitoring of medicinal products. In: Guidelines for good clinical practice (GCP) for trials on pharmaceutical products. Geneva, World Health Organization, 1995: Annex 3 (WHO Technical Report Series, No. 850).

3. WHO. Effective communications in pharmacovigilance: the Erice Report. Uppsala, World Health Organization Collaborating Centre for International Drug Monitoring,1998.

4. WHO. The World Health Organization Collaborating Centre for International Drug Monitoring [web site]. The World Health Organization Collaborating Centre for International Drug Monitoring, Uppsala (http://www.whoumc.org, accessed 15 October 2004).

5. Ellenberg SS, Fleming TR, DeMets DL. Data monitoring 
committees in clinical trials: a practical perspective. John Wiley \& Sons, 2002.

6. US National Institutes of Health. Further guidance on data and safety monitoring for phase I and II trials, OD-00-038, 5 June, 2000, http://grants1.nih.gov/grants/guide/noticefiles/NOT-OD-00-038.html, accessed 15 May 2006.

7. Hibberd PL, Weiner DL. Monitoring participant safety in phase I and II interventional trials: Options and controversies. Journal of Investigative Medicine 2004; 52:446-52.

8. Pocock SJ. Clinical trials: a practical approach. Chichester: Wiley, 1983 